Internat. J. Math. \& Math. Sci.

Vol. 24, No. 1 (2000) 67-71

S0161171200003185

(C) Hindawi Publishing Corp.

\title{
PERIODIC SOLUTIONS OF NONLINEAR DIFFERENTIAL EQUATIONS
}

\author{
XIAOJING YANG
}

(Received 26 March 1999)

\begin{abstract}
The periodic boundary value problems of a class of nonlinear differential equations are investigated.
\end{abstract}

Keywords and phrases. Periodic solutions, nonlinear equations, homeomorphism.

2000 Mathematics Subject Classification. Primary 34C25.

1. Introduction. Let us consider the following nonlinear differential equation

$$
\left(\bar{a}(t) \bar{\varphi}_{p}\left(x^{\prime}(t)\right)\right)^{\prime}+f(t, x(t))=0,
$$

where ' $=d / d t, f: \mathbb{R} \times \mathbb{R}^{n} \rightarrow \mathbb{R}^{n}$ is continuous, $2 \pi$-periodic in $t$ and $f(t, \cdot) \in \mathbb{C}^{1}$ $\left(\mathbb{R}^{n}, \mathbb{R}^{n}\right), \bar{a}(t) \bar{\varphi}_{p}(x)=: \operatorname{col}\left(a_{1}(t) \varphi_{p}\left(x_{1}\right), \ldots, a_{n}(t) \varphi_{p}\left(x_{n}\right)\right), a_{k}(t)$ is $2 \pi$-periodic and $a_{k}(t) \in \mathbb{C}^{1}(\mathbb{R},(0, \infty)), \varphi_{p}: \mathbb{R} \rightarrow \mathbb{R}$ be defined by $\varphi_{p}(s)=|s|^{p-2} s$, with $p>1$ fixed, $f(t, x)=\operatorname{col}\left(f_{1}(t, x), \ldots, f_{n}(t, x)\right)$.

When $p=2, a_{k}(t) \equiv 1, k=1,2, \ldots, n$. Equation (1.1) is of the form

$$
x^{\prime \prime}(t)+f(t, x)=0 .
$$

Amaral and Pera [1] and recently Li [5] proved the existence and uniqueness results of (1.2) under the following assumptions:

(L) There exist two constant symmetric $n \times n$ matrices $A_{0}$ and $B_{0}$ with eigenvalues $N_{k}^{2}$ and $\left(N_{k}+1\right)^{2},(k=1,2, \ldots, n)$, respectively, $N_{k} \geq 0$ is an integer for each $k, D_{2} f(t, x)$ is symmetric and there exist two time-dependent continuous symmetric $n \times n$ matrices $A(t)$ and $B(t)$ such that $A_{o} \leq A(t) \leq D_{2} f(t, x) \leq B(t) \leq B_{0}$ for all $(t, x) \in \mathbb{R} \times \mathbb{R}^{n}$. Furthermore, $N_{k}^{2}<\lambda_{k}(t) \leq \mu_{k}(t)<\left(N_{k}+1\right)^{2}$ on a subset of $[0,2 \pi]$ of positive measure, where $\lambda_{k}(t)$ and $\mu_{k}(t)$ are the eigenvalues of $A(t)$ and $B(t)$, respectively.

Inspired by the work of Li [5], we give sufficient conditions for the existence and uniqueness of the $2 \pi$-periodic solution of (1.1) by using the initial value problem method and homeomorphism of $\mathbb{R}^{n}$ to $\mathbb{R}^{n}$.

2. Initial value problem and eigenvalues problem. Throughout this paper, we denote the interval $[0,2 \pi]$ and $M_{n}$ denotes the set of all complex $n \times n$ matrices. We also assume the solutions of (1.1) exist on $I$ for any initial value $\left(x(0), x^{\prime}(0)\right) \in \mathbb{R}^{2 n}$.

Let us consider the initial value problem

$$
u^{\prime}(t)=g(t, u(t)), \quad u(0)=u_{0} .
$$


LEMmA $2.1[3,4]$. Assume that $g \in \mathbb{C}\left(I \times \mathbb{R}^{n}, \mathbb{R}^{n}\right)$ and possesses continuous partial derivatives $\partial g / \partial u$ on $I \times \mathbb{R}^{n}$. Let the solution $u_{0}(t)=u\left(t, t_{0}, u_{0}\right)$ of (2.1) exist for $t \in I$ and let

$$
H\left(t, t_{0}, u_{0}\right)=\frac{\partial g\left(t, u\left(t, t_{0}, u_{0}\right)\right)}{\partial u}
$$

Then

$$
\phi\left(t, t_{0}, u_{0}\right)=\frac{\partial u\left(t, t_{0}, u_{0}\right)}{\partial u_{0}}
$$

exists and is the solution of

$$
V^{\prime}=H\left(t, t_{0}, u_{0}\right) V
$$

such that $\phi\left(t_{0}, t_{0}, u_{0}\right)$ is the unit matrix.

LEMмA 2.2 [2]. Suppose $A \in M_{n}$. Then $\lambda$ is an eigenvalue of the matrix $A$ if and only if $\exp \lambda$ is an eigenvalue of the matrix $\exp A$.

LEMMA 2.3 [2]. If $A \in M_{n}$ and there exists $\delta>0$ such that $|\lambda|>\delta$ for all eigenvalues $\lambda$ of $A$, then $\left\|A^{-1}\right\| \leq \delta^{-n}\|A\|^{n-1}$, where $\|A\|=\max \lambda^{1 / 2}\left(A^{*} A\right)\left[A^{*}\right.$ denotes the adjoint of $A$, i.e., if $\left.A=\left(a_{i j}\right), A^{*}=\left(\bar{a}_{j i}\right)\right]$.

LEMMA 2.4 [7]. If $A \geq B \geq 0$, and $A$ and $B$ are two real symmetric $n \times n$ matrices, where $\lambda_{1} \leq \lambda_{2} \leq \cdots \leq \lambda_{n}$ and $\mu_{1} \leq \mu_{2} \leq \cdots \leq \mu_{n}$ are eigenvalues of $A$ and $B$, respectively, then $\lambda_{k} \geq \mu_{k}$, for $k=1,2, \ldots, n$.

LEMmA 2.5 [6]. Assume that $F: \mathbb{R}^{n} \rightarrow \mathbb{R}^{n}$ is continuously differentiable on $\mathbb{R}^{n}$ and $\left\|\left[F^{\prime}(x)\right]^{-1}\right\| \leq M<+\infty$ for all $x \in \mathbb{R}^{n}$. Then $F$ is a homeomorphism of $\mathbb{R}^{n}$ onto $\mathbb{R}^{n}$.

LEMmA 2.6. Assume $A, B$ are $n \times n$ matrices, then the eigenvalues of the $2 n \times 2 n$ matrix

$$
\left(\begin{array}{cc}
0 & \bar{A} \\
-\bar{B} & 0
\end{array}\right)
$$

are the roots of $\operatorname{det}\left(\lambda^{2} I_{n}+\overline{B A}\right)=0$.

Proof. From the following matrices equality

$$
\left(\begin{array}{cc}
\lambda I_{n} & 0 \\
-\bar{B} & \lambda I_{n}
\end{array}\right)\left(\begin{array}{cc}
\lambda I_{n} & -\bar{A} \\
\bar{B} & \lambda I_{n}
\end{array}\right)=\left(\begin{array}{cc}
\lambda^{2} I_{n} & -\lambda \bar{A} \\
0 & \lambda^{2} I_{n}+\overline{B A}
\end{array}\right)
$$

we obtain the result of Lemma 2.6 immediately.

3. Main results. Rewrite (1.1) as follows

$$
x^{\prime}=\varphi_{q}(b(t) y), \quad y^{\prime}=-f(t, x),
$$

where $b(t) y=: \operatorname{col}\left(b_{1}(t) y_{1}(t), \ldots, b_{n}(t) y_{n}(t)\right), b_{k}(t)=a_{k}^{-1}(t)$, and $(1 / p)+(1 / q)=1$, $(q=p /(p-1)), y_{k}(t)=a_{k}(t) \varphi_{p}\left(x_{k}^{\prime}\right)$, hence $x_{k}^{\prime}(t)=\varphi_{q}\left(b_{k}(t) y_{k}(t)\right), k=1,2, \ldots, n$. 
Let $u=\operatorname{col}(x, y) \in \mathbb{R}^{2 n}, g(t, u)=\operatorname{col}\left(\varphi_{q}(b(t) y),-f(t, x)\right) \in \mathbb{R}^{2 n}, v=\operatorname{col}(\alpha, \beta)=$ $\operatorname{col}(x(0), y(0))=\operatorname{col}\left(x(0), a(0) \varphi_{p}\left(x^{\prime}(0)\right)\right) \in \mathbb{R}^{2 n}$, then (3.1) is of the form

$$
u^{\prime}(t)=g(t, u(t)), \quad u(0)=v .
$$

Consider the variation equation of (3.2) with respect to $u$

$$
\xi^{\prime}=\frac{\partial g(t, u)}{\partial u} \xi
$$

where

$$
\frac{\partial g(t, u)}{\partial u}=\left(\begin{array}{cc}
0 & \frac{\partial \varphi_{q}(b(t) y)}{\partial y} \\
\frac{-\partial f(t, x)}{\partial x} & 0
\end{array}\right)=:\left(\begin{array}{cc}
0 & A \\
-B & 0
\end{array}\right)
$$

with

$$
\begin{gathered}
A=(q-1) \operatorname{diag}\left(b_{1}(t)\left|y_{1}(t)\right|^{q-2}, \ldots, b_{n}(t)\left|y_{n}(t)\right|^{q-2}\right), \\
B=\frac{\partial f(t, x(t))}{\partial x}=\nabla f(t, x(t)) .
\end{gathered}
$$

Let

$$
Z(t)=\exp \int_{0}^{t} \frac{\partial g(s, u(s, v))}{\partial u} d s .
$$

Then $Z(t)$ is a fundamental solution matrix of (3.3) and $Z(0)=I_{2 n}$. Meanwhile, by Lemma 2.1 we know that

$$
\frac{\partial u}{\partial v}=\left(\begin{array}{ll}
\frac{\partial x(t, v)}{\partial \alpha} & \frac{\partial x(t, v)}{\partial \beta} \\
\frac{\partial y(t, v)}{\partial \alpha} & \frac{\partial y(t, v)}{\partial \beta}
\end{array}\right)
$$

is also a fundamental solution matrix of (3.3). Therefore

$$
Z(t)=\frac{\partial u(t, v)}{\partial v}, \quad t \in[0,2 \pi] .
$$

Define: $h, H: \mathbb{R}^{2 n} \rightarrow \mathbb{R}^{2 n}, h(v)=\operatorname{col}(x(2 \pi, v), y(2 \pi, v))$,

$$
H(v)=v-h(v)
$$

By Lemma 2.1, $h(v)$ is $\mathbb{C}^{1}$-differentiable and so is $H(v)$. Therefore, solving periodic solution of (1.1) is equivalent to finding the fixed points of $h(v)$ or the zero points of $H(v)$. From (3.8) and (3.9)

$$
\begin{aligned}
H^{\prime}(v) & =I_{2 n}-h^{\prime}(v)=I_{2 n}-\frac{\partial u(2 \pi, v)}{\partial v}=I_{2 n}-Z(2 \pi) \\
& =I_{2 n}-\exp \int_{0}^{2 \pi} \frac{\partial g(t, u(t, v))}{\partial u} d t
\end{aligned}
$$


THEOREM 3.1. Let $\bar{A}_{v}=(q-1) \operatorname{diag}\left(\int_{0}^{2 \pi} b_{1}(t)\left|\beta_{1}-\int_{0}^{t} f_{1}(\tau, x(\tau)) d \tau\right|^{q-2} d t, \ldots\right.$, $\left.\int_{0}^{2 \pi} b_{n}(t)\left|\beta_{n}-\int_{0}^{t} f_{n}(x, x(\tau)) d \tau\right|^{q-2} d t\right), \bar{B}_{v}=\int_{0}^{2 \pi} \nabla f(t, x(t)) d t$, where $x(t)$ is any solution of (1.1) satisfying initial conditions $(x(0), y(0))=v=(\alpha, \beta) \in \mathbb{R}^{2 n}$. If there exist $v \in \mathbb{R}^{2 n}$ and integers $N_{k} \geq 0, k=1,2, \ldots, n$, such that the matrix $\bar{B}_{v} \bar{A}_{v}$ is similar to a diagonal matrix $C_{v}=\operatorname{diag}\left(\lambda_{1}, \ldots, \lambda_{n}\right)$ with $\left(2 \pi N_{k}\right)^{2}<\lambda_{k}<\left[2 \pi\left(N_{k}+1\right)\right]^{2}, k=$ $1,2, \ldots, n$. Then (1.1) has a unique $2 \pi$-periodic solution $x(t)$ satisfying the initial condition $(x(0), y(0))=v$.

Proof. By Lemma 2.5, we need only to show that $H^{\prime}(V)$ is invertible and that there exists a constant $M>0$ such that $\left\|\left[H^{\prime}(v)\right]^{-1}\right\| \leq M$.

In fact, from (3.1) and (3.5), $A=(q-1) \operatorname{diag}\left(b_{1}(t)\left|y_{1}(t)\right|^{q-2}, \ldots, b_{n}(t)\left|y_{n}(t)\right|^{q-2}\right)$, since

$$
y_{k}(t)=y_{k}(0)-\int_{0}^{t} f_{k}(\tau, x(\tau)) d \tau=\beta_{k}-\int_{0}^{t} f_{k}(\tau, x(\tau)) d \tau, \quad k=1,2, \ldots, n
$$

we have

$$
\begin{aligned}
\bar{A}_{v}=\int_{0}^{2 \pi} A d t=(q-1) \operatorname{diag} & \left(\int_{0}^{2 \pi} b_{1}(t)\left|\beta_{1}-\int_{0}^{t} f_{1}(\tau, x(\tau)) d \tau\right|^{q-2} d t, \ldots,\right. \\
& \left.\times \int_{0}^{2 \pi} b_{n}(t)\left|\beta_{n}-\int_{0}^{t} f_{n}(\tau, x(\tau)) d \tau\right|^{q-2} d t\right) .
\end{aligned}
$$

From Lemma 2.6, the eigenvalues of the matrix $\left(\begin{array}{cc}0 & \bar{A}_{v} \\ -\bar{B}_{v} & 0\end{array}\right)$ are $\pm \sqrt{\lambda_{1}} i, \pm \sqrt{\lambda_{2}} i, \ldots, \pm \sqrt{\lambda_{n}} i$. By (3.5), (3.10), and Lemma 2.2 the eigenvalues of $H^{\prime}(v)$ are

$$
\mu_{k}=1-\exp \left( \pm \sqrt{\lambda_{k}} i\right)=1-\cos \sqrt{\lambda_{k}} \mp i \sin \sqrt{\lambda_{k}}, \quad k=1,2, \ldots, n .
$$

From the assumption of $\lambda_{k}$ and

$$
\left|\mu_{k}\right|=\sqrt{2-2 \cos \sqrt{\lambda_{k}}}=2\left|\sin \frac{\sqrt{\lambda_{k}}}{2}\right|
$$

it follows that

$$
\left|\mu_{k}\right| \geq 2 \min _{1 \leq k \leq n}\left(\left|\sin \frac{\sqrt{\lambda_{k}}}{2}\right|\right)>0
$$

because $N_{k} \pi<\sqrt{\lambda_{k}} / 2<\left(N_{k}+1\right) \pi, k=1,2, \ldots, n$,

$$
\left\|\left[H^{\prime}(v)\right]^{-1}\right\| \leq \frac{1+\exp \left(4 \max _{1 \leq k \leq n}\left(N_{k}+1\right)^{2} \pi^{2}\right)}{\left(2 \min \left\{\left|\sin \sqrt{\lambda_{k}} / 2\right|\right\}\right)^{n}}=M .
$$

Now, from Lemma 2.5, $H^{\prime}(v)$ is invertible, since by Lemma $2.3, H^{\prime}(v)$ is homeomorphism of $\mathbb{R}^{n}$ onto $\mathbb{R}^{n}$, hence there exists a unique $v_{0} \in \mathbb{R}^{n}$ such that $H\left(v_{0}\right)=0$, that is, $h\left(v_{0}\right)=v_{0}$. Theorem 3.1 is proved.

COROLlary 3.2. Let $p=2, a_{k}(t) \equiv 1, k=1,2, \ldots, n$ in (1.1), and suppose ( $L$ ) holds, then (1.1) has a unique $2 \pi$-periodic solution. 
Proof. In this case, $q=2$, hence $\bar{A}=2 \pi I_{n}$, then eigenvalues of $\left(\begin{array}{cc}0 & \bar{A} \\ -\bar{B} & 0\end{array}\right)$ are

$$
\pm \sqrt{2 \pi \lambda_{k}} i, \quad k=1,2, \ldots, n
$$

with

$$
2 \pi N_{k}^{2}<\lambda_{k}<2 \pi\left(N_{k}+1\right)^{2},
$$

therefore

$$
\left\|\left[H^{\prime}(v)\right]^{-1}\right\| \leq \frac{1+\exp \left(2 \max _{1 \leq k \leq N}\left(N_{k}+1\right)^{2} \pi\right)}{\left(2 \min _{1 \leq k \leq N}\left\{\sin \left|\sqrt{2 \pi a_{k}} / 2\right|, \sin \left|\sqrt{2 \pi b_{k}} / 2\right|\right\}\right)^{2}}=M,
$$

where

$$
2 \pi N_{k}^{2}<a_{k}=\int_{0}^{2 \pi} \lambda_{k}(t) d t \leq \int_{0}^{2 \pi} \mu_{k}(t) d t=b_{k}<2 \pi\left(N_{k}+1\right)^{2} .
$$

From Lemma 2.3 again, (1.1) has a unique $2 \pi$-periodic solution. Corollary 3.2 is proved.

REMARK 3.3. Corollary 3.2 is the result of [1] and [5].

\section{REFERENCES}

[1] L. Amaral and M. P. Pera, On periodic solutions of nonconservative systems, Nonlinear Anal. 6 (1982), no. 7, 733-743. MR 84k:58063. Zbl 532.47052.

[2] K. J. Brown, Nonlinear boundary value problems and a global inverse function theorem, Ann. Mat. Pura Appl. (4) 106 (1975), 205-217. MR 53\#6375. Zbl 326.35021.

[3] V. Lakshmikantham and S. Leela, Differential and Integral Inequalities: Theory and Applications. Vol. I: Ordinary Differential Equations, Academic Press, New York, 1969. MR 52\#837. Zbl 177.12403.

[4] _ Differential and Integral Inequalities: Theory and Applications. Vol. II: Functional, Partial, Abstract, and Complex Differential Equations, Academic Press, New York, 1969. MR 52\#838.

[5] W. Li, Solving the periodic boundary value problem with the initial value problem method, J. Math. Anal. Appl. 226 (1998), no. 1, 259-270. MR 99h:34033. Zbl 911.34017.

[6] J. M. Ortega and W. C. Rheinboldt, Iterative Solutions of Nonlinear Equations in Several Variables, Academic Press, New York, 1970. MR 42\#8686. Zbl 241.65046.

[7] J. G. Sun, Matrix Perturbed Analysis, Academic Press, Beijing, 1987 (Chinese).

Xiaojing YAng: Department of Mathematics, Tsinghua University, Beijing, China 


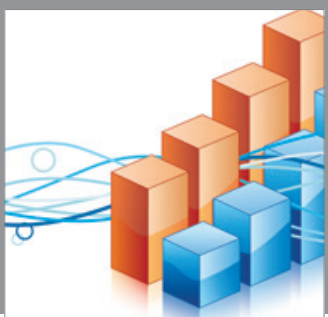

Advances in

Operations Research

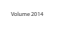

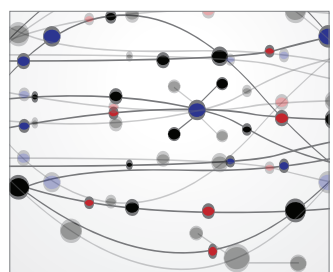

\section{The Scientific} World Journal
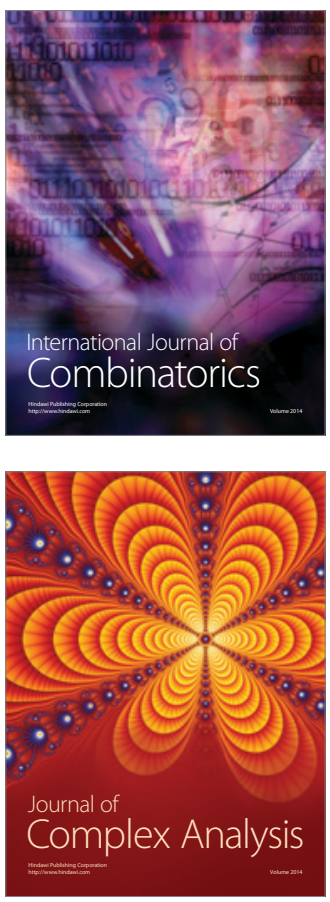

International Journal of

Mathematics and

Mathematical

Sciences
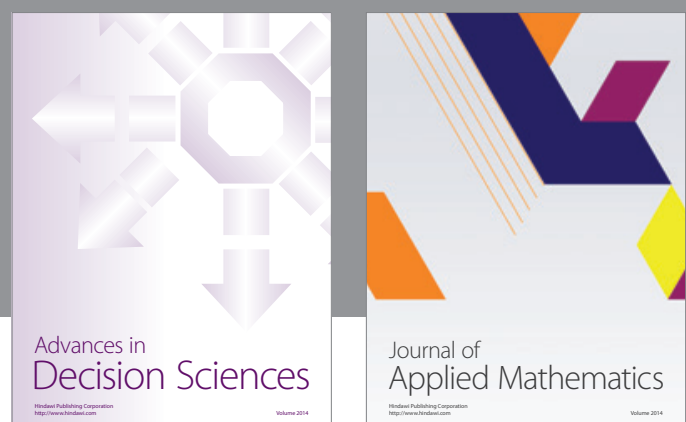

Journal of

Applied Mathematics
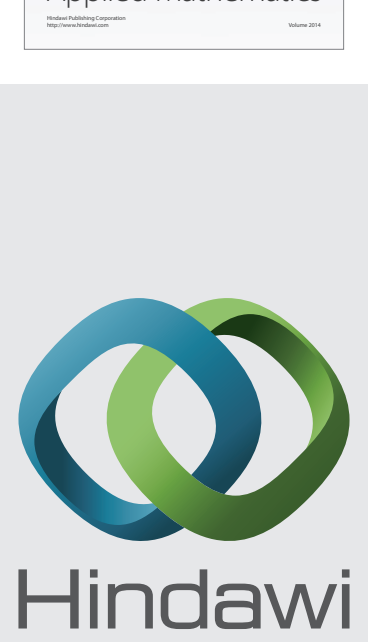

Submit your manuscripts at http://www.hindawi.com
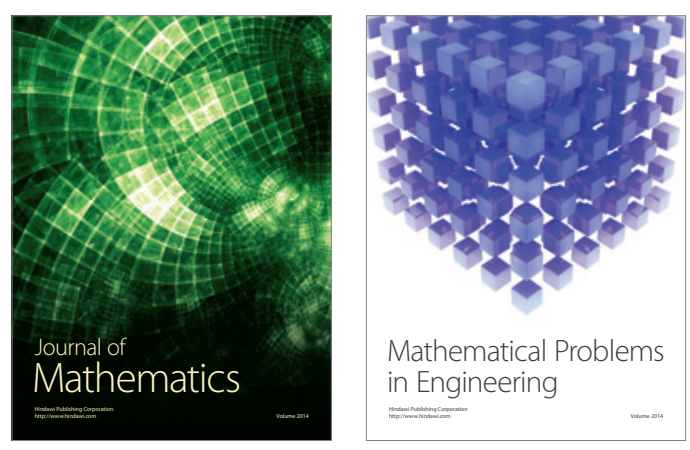

Mathematical Problems in Engineering
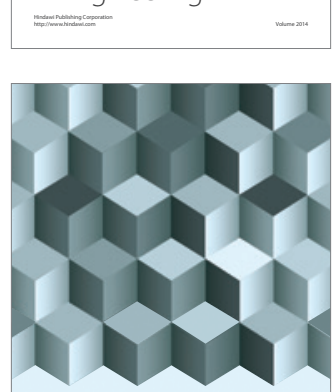

Journal of

Function Spaces
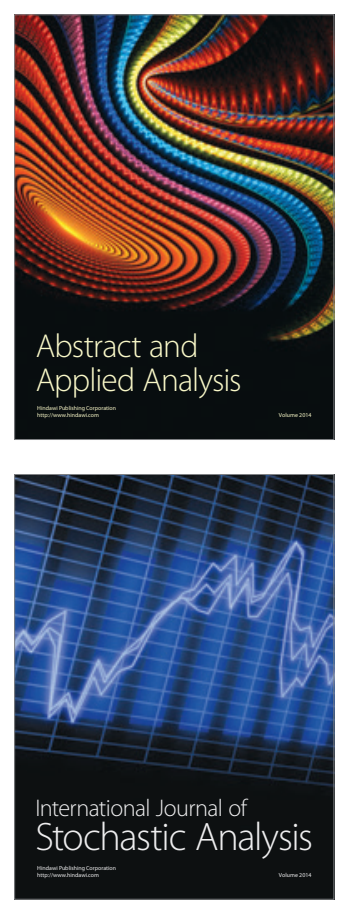

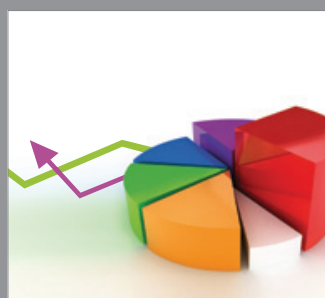

ournal of

Probability and Statistics

Promensencen
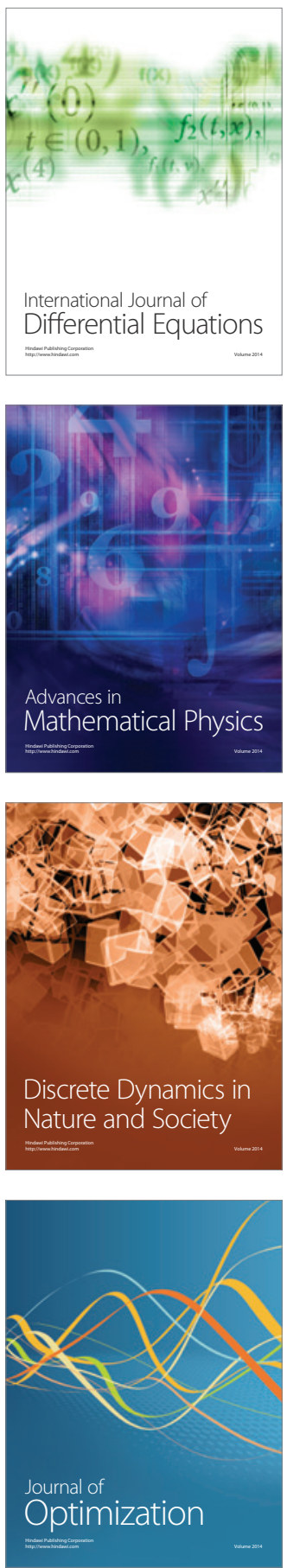